\title{
THE ESTIMATION OF CONTROL SYSTEMS STABILITY BOUNDARIES BY THE DESCRIBING FUNCTION METHOD BY EXAMPLE OF AIRCRAFT
}

\author{
Iuliia Zaitceva \\ Institute for Problems in \\ Mechanical Engineering of the RAS, \\ Russia \\ juliazaytsev@gmail.com
}

\author{
Leonid Chechurin \\ Department of Industrial \\ Engineering and Management, \\ LUT University, \\ Finland \\ Leonid.Chechurin@lut.fi
}

Article history:

Received 06.03.2020, Accepted 14.09.2020

\begin{abstract}
In this paper, the conditions for periodic modes formation in the closed-loop pilot-vehicle system in the compensatory control mode are established. The case of high gain pilot task is considered under the conditions of a sudden disturbance, as well as under the influence of the system nonlinearities, such as actuator position and rate limit. Sinusoidal input describing function method and parametric resonance equation were used to determine the onset conditions for self-oscillations and forced oscillations. The case when this methods lead to erroneous results is established. The numerical limits of permissible pilot gain, time delay and reference signal at which unstable periodic modes do not arise are calculated.
\end{abstract}

\section{Key words}

nonlinear, describing function, PIO, aircraft, time delay, self-oscillations, forced oscillations.

\section{Introduction}

Modern flight control systems have a complex configuration, a variety of targets and must meet the requirements of high reliability and safety. Despite high level of automation, aircraft control remains manual using elevators and pedals. A human pilot is still an integral part of an aircraft and is closely connected with the flight control loop. In this regard, there is a need to study the effective dynamics of an aircraft in various flight modes.

The emergence of oscillatory modes is always undesirable and dangerous for an aircraft. When controlling in a closed loop, the so-called pilot-induced oscillations (PIO) are isolated. They are characterized by a sudden appearance of aircraft oscillations in the longitudinal and/or lateral direction with a small or jump-resonant amplitude. The reasons for these oscillations are different and explained by several factors. Typically, oscillations excitation triggers alter the dynamics component of a closed-loop pilot-vehicle system, resulting in an instability or weakly damped system. Thus, effective aircraft dynamics with a high gain task is a necessary conditions for PIO. appearance is the presence of effective aircraft dynamics with a high gain task. Tracking tasks with high pilot gain are characterized by precise control, quick reactions and the desire for small errors typical during aerial refueling, formation flying and carrier approach [McRuer and Warner, 1997; Anderson, 2005].

PIO often occurs under unusual flight conditions caused by a sudden change in environmental conditions, which in turn changes pilot dynamics. For example, when an unexpected disturbance arises, leading to a change in the overloads and orientation angles of the aircraft and pilot control with some time delay. If external disturbance source is unknown for the pilot, they intervene in the control with delay and the stabilization process takes several seconds introducing a significant negative phase shift between the input and output signal [Byushgens and Studnev, 1979; McRuer et al., 1973]. Nonlinearities in the aircraft control actuator are considered to be one of the identified causes of PIO. They arise due to physical constraints of stickkolumn deflections, actuators position and rate limiters, and limiters in the controller software.

In fly-by-wire system, the cockpit control device cannot transmit information about saturation to the pilot. As a result, the pilot can issue ill-timed or/and overshoot commands. In this regard, several studies identify the oscillatory modes of nonlinear pilot-vehicle systems. One of the widely used methods for studying such systems is the numerical-analytical harmonic balance method, 
or sinusoidal input describing function (SIDF) [Garber and Rozenvasser, 1965; Leonov and Kuznetsov, 2011; Leonov and Kuznetsov, 2013]. With this method a nonlinear element is replaced with a linear one, which converts harmonic oscillations in the same way as a nonlinear element and is characterized by an equivalent complex gain. Such a replacement allows studying of nonlinear systems by frequency methods. In particular, with frequency methods, it is possible to detect the presence of self-oscillations, investigate their stability, determine their amplitude and frequency, and solve the problems of a nonlinear system correction [Alcala et al., 2004].

Thus, in an asymptotically stable system [Andrievsky et al., 2020; Kuznetsov, 2020; Andrievsky et al., 2013; Andrievsky et al., 2016], the excitation conditions for hidden oscillations were revealed. The pilot gain value for autonomous motion was numerically determined, the stability boundaries of the system when it changes were shown on the Nyquist plot, and the value of the required reference input for the forced movement was determined. Attention to the effects of rate limiting and other system nonlinearities was given after severe PIO events with the YF-12 aircraft during aerial refueling. SIDF method for a rate limited actuator and an inverse describing function method were used to predict limit cycle oscillations [Ashkenas et al., 1964; Klyde et al., 1995]. It was examined the effects of the simultaneous presence of position and rate saturation in the control loop and the value of the input signal causing the limit cycles have been established [Amato et al., 2000]. Here the effectiveness of the SIDF method was confirmed during the X-15 flight, but the paper does not present numerical results and confirmatory modelling.

In all cases, where aircraft dynamics are changing rapidly, it is important to know how quickly the pilot can adapt to the changing characteristics of the aircraft. Evaluation of the numerical parameters of the system in such cases is important to form requirements for the degree of redundancy of automatic devices. In this regard, this paper proposes to find the boundaries of the system parameters at which the processes in the system lose stability. A more detailed consideration of the case with actuator rate limit in the frequency domain is presented finding reasons why the SIDF method gives incorrect results in the study of pilot-aircraft systems.

\section{The SIDF Method and Equation of Parametric Resonance}

SIDF is a method that allows to extend the transfer function concept of linear system studies to the nonlinear system problem [Garber and Rozenvasser, 1965]. It is the SIDF method to replace a system nonlinearity by a linear gain, which renders similar the responses of the nonlinearity and its approximation. When using the SIDF method for the nonlinear dynamical system analysis, the assumptions are made that the linear part of the system does not pass high frequencies and the phase balance is observed. Consider the equations of harmonic balance for self-oscillations and forced oscillations. Following [Krylov and Bogolyubov, 1947; Goldfarb et al., 1976; Lurie and Enright, 2020], we assume that a harmonic signal is fed to the input of the nonlinear element $F(u)$ :

$$
u(t)=A \sin \psi,
$$

where phase $\psi=\omega t, \omega$ is frequency, $t$ is time. Then the output periodic signal of the nonlinear element takes the form $y(t)=F(A \sin \omega t)$ and can be expanded in Fourier series, where the harmonic linearization coefficients for the odd-symmetric nonlinear function of the first harmonic amplitude $A$ to the $m$-th harmonic amplitude in complex form are:

$$
W_{m}(A)=\frac{1}{\pi A} \int_{0}^{2 \pi} F(A \sin \psi) e^{-j m \psi} d \psi,
$$

where $m$ is harmonic number. Equation (2) gives a SIDF, which is approximately equivalent to the transfer function of the nonlinearity. Such a representation of nonlinearity allows one to study the system as linear, including the study of limit cycles.

For an autonomous dynamic system containing the linear part $W(j \omega)$ and nonlinear in the form of a harmonically linearized element with respect to the first harmonic $W_{1}(A)$ the harmonic balance equation has the form:

$$
W^{-1}(j \omega)+W_{1}(A)=0
$$

For non-autonomous system, when forced oscillations act with amplitude $A_{i}$, the condition of harmonic balance is written in the form:

$$
W^{-1}(j \omega)+W_{1}(A)=\frac{A_{i}}{A} e^{-j \varphi},
$$

where $\varphi$ is phase shift between output and reference signal. When considering the influence of higher harmonics, the same reasoning is used as for the first approximation.

To solve the general problem of stability consider a nonlinear non-autonomous system in the form:

$$
\dot{x}(t)=f(x(t), t),
$$

where $f[\cdot]$ is nonlinear vector-function. Suppose that there exists a solution to this system $u^{*}(t)$ and perturbed motion in the form:

$$
u(t)=u^{*}(t)+\Delta u(t) .
$$

Substituting the equation (6) in (5), we obtain the equation in increments, in which nonlinearity is

$$
\left.\frac{\Delta F\left[u^{*}(t)+\Delta u(t)\right]}{\Delta u(t)} \Delta u(t)\right|_{\Delta u(t) \rightarrow 0}=\frac{d F(u)}{d u}
$$




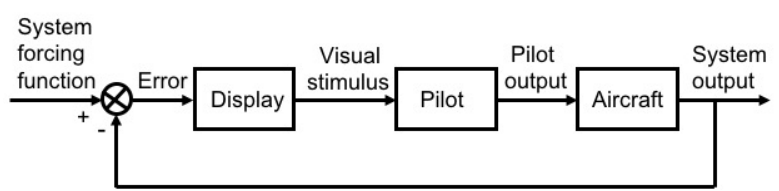

Figure 1. Simple compensatory system [McRuer, 1995]

If the equation in increments has a stable single state of equilibrium, then the unperturbed motion of the original equation is also stable. The derivative of the nonlinear element $d F / d u$, calculated along the periodic solution of the equation (5), is a periodically changing parameter. The associated system oscillations are called parametric oscillations, which can lead to the phenomenon of parametric resonance and, in turn, loss of stability system. For stability analysis, $F(u)$ is presented in a stationary form (2). The condition for parametric resonance excitation in a nonlinear system is written as [Chechurin and Chechurin, 2017]:

$$
W^{-1}(j \omega)=a_{0}+\rho e^{-j \phi},
$$

where $a_{0}=-W_{1}(A)-\frac{A}{2} \frac{d W_{1}(A)}{d A}, \rho=-\frac{A}{2} \frac{d W_{1}(A)}{d A}$ are the center and circle radius of parametric excitation, respectively, $\phi$ is the phase shift between the periodic parameter oscillations and the input signal.

To clarify the stability conditions for forced oscillations, we consider the formation conditions of forced oscillations (4) and the condition for the parametric resonance excitation (8) together. These equations do not have solution if the diameter of the circumference of the excitation of parametric resonance is less than the radius of the circumference of the forced oscillations. Then the stability condition of the forced oscillations is written as:

$$
A_{i}>A^{2}\left|\frac{d W_{m}(A)}{d A}+W^{-1}(j \omega)\right|
$$

Thus, the above equations will be used to study the nonlinear pilot-vehicle system.

\section{Pilot-vehicle system model}

Consider a model of a flight control system along a longitudinal channel in the compensatory movement mode. Longitudinal movement is fundamental at all essential stages of a flight, such as take-off, climbing, cruising, landing and maneuvering in a vertical plane. In this mode, commands and interference are generated randomly, and the pilot acts based on system errors or aircraft output. In conditions of full attention, the pilot performs continuous control in a closed loop to minimize the stabilization error. Notice that the system output follows the system forcing function command to the closed-loop pilot-vehicle system quite closely. Since the dynamics of the real actuator are nonlinear, we take into account active nonlinearities, such as position and rate limit, to study the system. The model is graphically shown in Fig. 1.

The pilot model is usually presented in the form of a series-connected static element acting on the pitch error signal [MeRuer et al., 1965; Byushgens and Studnev, 1979; McRuer, 1995; Efremov et al., 2015]. During disturbance compensation, the pilot acts as a proportional or relay link, striving for control simplicity, and then the pilot model is represented as a static coefficient with delay:

$$
W_{p}(s)=K_{p} e^{-\tau s},
$$

where $K_{p}$ is pilot gain, $\tau$ is pilot time delay.

The aircraft under investigation is controlled remotely through a joystick, which acts as an elevator. The aircraft actuator is modelled by an aperiodic link of the first order [Andrievsky et al., 2013]. In this paper, the following aircraft model is taken as an example, where the transfer function from the elevator's angle of deviation $\delta_{e}$ to the pitch angle $\vartheta$ has the form [Mandal and $\mathrm{Gu}, 2016$ ]:

$$
W_{a}(s)=\frac{30 s^{2}+115 s-49.3}{s^{4}+7 s^{3}+23 s^{2}-10.6 s+0.3}
$$

It should be noted that this model is unstable and is stabilized by introducing the pitch rate feedback loop as follows: $u_{c}(t)=K_{p} e^{-\tau s} \varepsilon(t)-K_{q} q(t)$, where $u_{c}(t)$ is pilot signal, $\varepsilon$ is pitch tracking error, $q(t)$ is pitch rate, $K_{q}$ is rate gyro gain found by pole locus technique [Andrievsky et al., 2015].

\section{Oscillations Modes Stability}

In the previous work [Zaitceva and Chechurin, 2019] it was found that the study of actuator rate limit in pilotvehicle system by the SIDF method leads to erroneous results, while the calculation of the oscillations modes for the backlash is correct. After literature analysis of relay systems with internal feedback [Korolev, 1956; Fridman et al., 1993], it was revealed that the same nonlinearity in different places of the control loop behaves differently. Therefore, if nonlinearity is covered by a feedback loop, then nonlinearity function is no longer characterised by a static Fourier coefficient, but a resonant filter, which amplitude characteristics depend on the frequency.

Rate limit actuator nonlinearity is closed by feedback loop. The amplitude-frequency responses of rate limited actuator and position limited actuator with the same parameters are shown in Fig. 2 (under the ratio characterizing the slope of the nonlinear saturation function $\mathrm{K} / \mathrm{b}$ $=2$.)

The larger the signal amplitude at the nonlinearity input with respect to the actuator rate value, the higher the peak response.

As another example, consider a system (10), (11) with actuator position limit in the angle channel and accept $K / b=2$. Assume that the system forcing function 


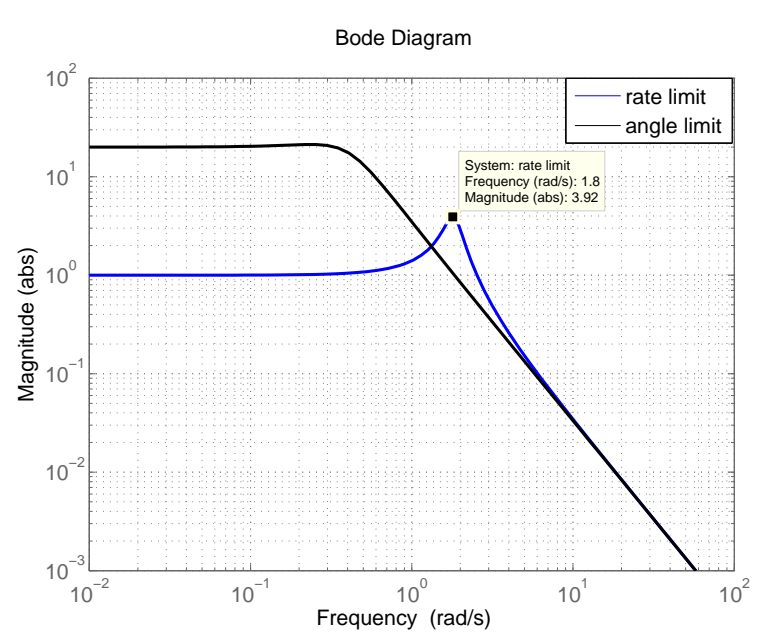

Figure 2. Amplitude-frequency response of rate and position limited actuator

changes in harmonic law (1) with the amplitude $A_{i}$ and the frequency $\omega$. The harmonic linearization coefficient of the saturation nonlinearity $q_{1}^{\prime}$ in accordance with (2) is:

$$
q_{1}^{\prime}=\frac{2 K}{\pi}\left[\arcsin \frac{b}{A}+\frac{b}{A} \sqrt{1-\left(\frac{b}{A}\right)^{2}}\right]
$$

According to (8), parametric excitation center and radius are:

$$
a_{0}=\frac{2 K}{\pi} \arcsin \frac{b}{A}, \quad \rho=\frac{2 K}{\pi}\left[\frac{b}{A} \sqrt{1-\frac{b}{A}}\right]
$$

Equation (4) has several solutions for various system parameters. One of them exists when under $K_{p}=2$, $\tau=0.1$, shown in Fig. 3. The parametric resonance circle is highlighted in red.

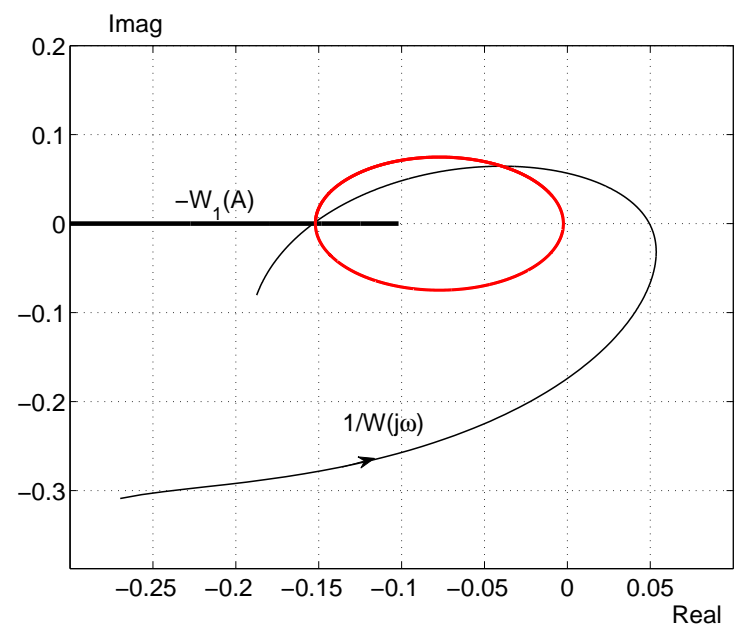

The intersection points of $W_{1}(A)$ and $W^{-1}(j \omega)$ hodographs have values: $\omega=13.8, A=16.5$. According to (9), forced oscillations are stable at $A_{i}>0.5$. Simulation result for reference $\vartheta$ and actual $\vartheta_{c}$ pitch angles is shown in Fig. 4. According to Fig. $1 \vartheta=A \sin (\omega t)$ is system forcing function, $\vartheta_{c}$ is system output. The process is stable and has amplitude attenuation due to the fact that the Fourier coefficient has an amplitude gain of less than one.

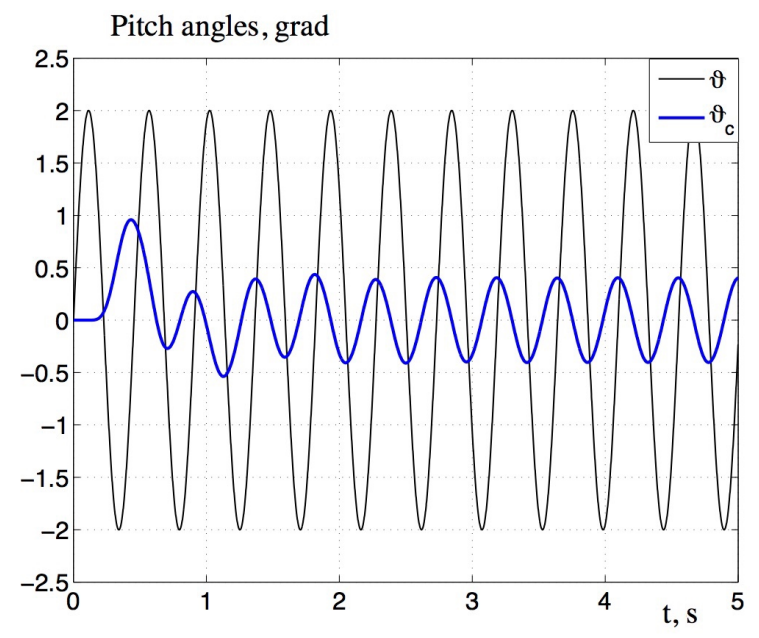

Figure 4. Pilot-vehicle system response for a sinusoidal input

Calculations showed that there is no solution of (4) for the Fourier coefficient of the high harmonics. Amplitude estimation of high harmonics showed that the SIDF method can be applied in terms of the first harmonic approximation.

\section{Conclusion}

The paper considers the closed-loop pilot-system in the compensatory control mode. It was found that in this system, it is possible to calculate the conditions for periodic modes formation using the SIDF method and parametric resonance equations for such nonlinearities as position limitation, backlash. The amplitude and frequency of self-oscillations were determined for position limitation nonlinearity, the amplitudes boundaries of the input signals were determined numerically in the first harmonic approximation, at which the forced oscillations are stable. Oscillatory modes caused by higher harmonics were not detected. Possible application of the work results will be useful for engineers investigating the nonlinear systems.

Figure 3. Harmonic balance 


\section{Acknowledgements}

Iuliia Zaitceva thanks Prof. Boris Andrievsky for valuable advice and recommendations. This work was financially supported by the Leading Scientific Schools of the Russian Federation program for 2020-2021 (SS2624.2020.1).

\section{References}

Alcala, I., Gordillo, F., and Aracil, J. (2004). Phase compensation design for prevention of pio due to actuator rate saturation. In Proc. American Control Conf. (ACC 2004), Boston, Massachusetts, USA, AACC, June 30July 2, pp. 4686-4691.

Amato, F., Iervolino, S., Pandit, M., Scala, S., and Verde, L. (2000). Analysis of pilot-in-the-loop oscillations due to position and rate saturations. In Proc. 39th IEEE Conf. on Decision and Control (CDC 2000), Sydney, Australia, IEEE, pp. 3564-3569.

Anderson, J. (2005). Fundamentals of aerodynamics. McGraw-Hill.

Andrievsky, B., Kravchuk, K., Kuznetsov, N., Kuznetsova, O., and Leonov, G. (2016). Hidden oscillations in the closed-loop aircraft-pilot system and their prevention. IFAC-PapersOnLine, 49, pp. 30-35.

Andrievsky, B., Kudryashova, E., Kuznetsov, N., and Kuznetsova, O. (2020). Aircraft wing rock oscillations suppression by simple adaptive control. Aerospace Science and Technology, 105, pp. 106049.

Andrievsky, B., Kuznetsov, N., Kuznetsova, O., Leonov, G., and Seledzhi, S. (2015). Nonlinear phase shift compensator for Pilot-Induced Oscillation prevention. In Prepr. 9th IEEE Europ. Modeling Symp. on Mathematical Modeling and Computer Simulation (EMS 2015), Madrid, Spain, 6 - 8 October, pp. 225-231.

Andrievsky, B., Kuznetsov, N., Leonov, G., and Pogromsky, A. (2013). Hidden oscillations in aircraft flight control system with input saturation. In Proc. 5th IFAC Intern. Workshop on Periodic Control Systems (PSYCO 2013), IFAC Proceedings Volumes (IFACPapersOnline), vol. 5(1), Caen, France, pp. 75-79.

Ashkenas, I. L., Jex, H. R., and McRuer, D. T. (1964). Pilot-induced oscillations: their cause and analysis. Technical Report 64-143, Northrop-Norair. Prepared by Systems Technology, Inc. Report STI TR-239-2.

Byushgens, G. S. and Studnev, R. V. (1979). Aircraft Aerodynamics: Dynamics of longitudinal and lateral movement. Mashinostroenie Publ., Moscow. (in Russian).

Chechurin, L. and Chechurin, S. (2017). Physical Fundamentals of Oscillations. Frequency Analysis of Periodic Motion Stability. Springer.

Efremov, A., Koshelenko, A., Tyaglik, M., Tyumencev, Y., and Wenqian, T. (2015). Mathematical modeling of pilot control response characteristics in studying the manual control tasks. Russian Aeronautics, $\mathbf{5 8}(2)$, pp. 173-179.
Fridman, L., Fridman, E., and Shustin, E. (1993). Steady modes in an autonomous system with break and delay. Differential Equations, 29(8), pp. 1161-1166.

Garber, E. D. and Rozenvasser, E. N. (1965). On studies of periodical regimes of non-linear systems on the basis of filter hypothesis. Automation and Remote Control, 26 (2), pp. 274-284.

Goldfarb, L. S., Baltrushevich, A. V., and Netushil, A. V. (1976). Theory of automatic control. Vysshaya shkola Publ., Moscow. (in Russian).

Klyde, D., McCruer, D., and Myers, T. (1995). Unified Pilot-Induced Oscillation Theory. Volume 1. PIO Analysis with Linear and Nonlinear Effective Vehicle Characteristics, Including Rate Limiting. Defense Technical Information Center.

Korolev, N. A. (1956). On periodic modes in relay systems with internal feedback. Avtomatika i Telemekhanika, 17 (11), pp. 969-978. (in Russian).

Krylov, N. and Bogolyubov, N. (1947). Introduction to nonlinear mechanics. In Annals of Mathematics Studies, vol. 11. Princeton University Press.

Kuznetsov, N. V. (2020). Theory of hidden oscillations and stability of control systems. Journal of Computer and Systems Sciences International, 59(5), pp. 647668.

Leonov, G. A. and Kuznetsov, G. V. (2013). Hidden attractors in dynamical systems. From hidden oscillations in Hilbert-Kolmogorov, Aizerman, and Kalman problems to hidden chaotic attractors in Chua circuits. Int J. Bifurcation and Chaos, 23 (1), pp. 1-69.

Leonov, G. A. and Kuznetsov, N. V. (2011). Analyticalnumerical methods for investigation of hidden oscillations in nonlinear control systems. IFAC Proceedings Volumes (IFAC-PapersOnline), 18 (1), pp. 2494-2505.

Lurie, B. and Enright, P. (2020). Describing function. In Classical Feedback Control with Nonlinear MultiLoop Systems. Boca Raton: CRC Press.

Mandal, T. and Gu, Y. (2016). Analysis of pilot-inducedoscillation and pilot vehicle system stability using UAS flight experiments. Aerospace, 3 (42), pp. 1-23.

McRuer, D. T. (1995). Pilot-Induced Oscillations and human dynamic behavior. Technical Report 4683, NASA, Hawthorne, CA, USA.

McRuer, D. T., Ashkenas, I., and Graham, D. (1973). Aircraft dynamics and automatic control. Princeton, N. J.

McRuer, D. T. and Warner, J. D., editors (1997). Aviation Safety and Pilot Control: Understanding and Preventing Unfavorable Pilot-Vehicle Interactions. Committee on the Effects of Aircraft-Pilot Coupling on Flight Safety Aeronautics and Space Engineering Board Commission on Engineering and Technical Systems National Research Council National Academy Press, Washington, DC.

MeRuer, D., Graham, D., Krendel, E., and Reisener, Jr., W. (1965). Human pilot dynamics in compensatory systems-theory, models, and experiments with con- 
trolled element and forcing function variations. Technical Report AFFDL-TR-65-15, Franklin Inst.

Zaitceva, I. S. and Chechurin, L. S. (2019). Stability of forced oscillations in manned aircraft systems. Differential equations and control processes, pp. 159-142. (in Russian). 